\title{
Change and clinical significance of serum cortisol, BNP, and PGE-2 levels in premature infants with patent ductus arteriosus
}

\author{
Qintao Cui, Xiaochen Liu, Guobao Su, Chaoyuan Zhou, Junhua Wang \\ Cardiovascular Surgery, the First Affiliated Hospital of Xinxiang Medical University, Xinxiang, China \\ Contributions: (I) Conception and design: Q Cui, X Liu; (II) Administrative support: J Wang; (III) Provision of study materials or patients: X Liu, G \\ Su; (IV) Collection and assembly of data: Q Cui, C Zhou; (V) Data analysis and interpretation: Q Cui, X Liu, G Su; (VI) Manuscript writing: All \\ authors; (VII) Final approval of manuscript: All authors. \\ Correspondence to: Qintao Cui. Cardiovascular Surgery, the First Affiliated Hospital of Xinxiang Medical University, No. 88 Weihui Health Road, \\ Xinxiang, China. Email: cui20070611@163.com.
}

Background: To analyze the change and clinical significance of cortisol, B-type brain natriuretic peptide (BNP), and prostacyclin-2 (PGE-2) levels in premature infants with patent ductus arteriosus (PDA).

Methods: A total of 67 cases of premature infants admitted to our hospital from January 2018 to April 2020 were included, all of whom developed PDA (PDA group). According to the presence or absence of symptoms, they were divided into the symptomatic group (28 cases) and the asymptomatic group (39 cases). In addition, 62 premature infants without PDA who were born in our hospital during the same period were selected as the control group. The expression levels of cortisol, BNP, and PGE-2 in infants in different groups and between infants with symptoms and without symptoms were analyzed, along with the risk factors leading to PDA in preterm infants. The value of cortisol, BNP, and PGE-2 in the diagnosis of PDA in premature infants was also analyzed.

Results: Compared with the control group, cortisol in the PDA group was significantly decreased $(\mathrm{P}<0.05)$, while the levels of $\mathrm{BNP}$ and $\mathrm{PGE}-2$ were significantly increased $(\mathrm{P}<0.05)$. The cortisol level in the asymptomatic group was significantly higher than that in the symptomatic group, while the levels of BNP and PGE-2 were opposite, and the differences were statistically significant $(\mathrm{P}<0.05)$. Logistic regression analysis showed that birth weight $<1,200 \mathrm{~g}$, decreased cortisol, increased BNP, and increased PGE-2 were independent risk factors leading to PDA in preterm infants, and the differences were statistically significant $(\mathrm{P}<0.05)$. Receiver operating characteristic (ROC) curve showed that the sensitivity and specificity of cortisol+BNP+PGE-2 in the diagnosis of PDA in premature infants were $75.60 \%$ and $73.10 \%$, respectively. The area under the curve (AUC) value was 0.759 (95\% CI: 0.611-0.859), which was significantly higher than the AUC values of the 3 tests alone $(\mathrm{P}<0.05)$.

Conclusions: The expression of cortisol decreased in premature infants with PDA, while the levels of BNP and PGE-2 significantly increased. Dynamic detection of the changes in these levels can provide an important reference for the early diagnosis of PDA and for the assessment of disease progression.

Keywords: Cortisol; B-type brain natriuretic peptide (BNP); prostacyclin-2 (PGE-2); patent ductus arteriosus in premature infants (PDA in premature infants); clinical significance

Submitted Aug 25, 2021. Accepted for publication Oct 09, 2021.

doi: $10.21037 /$ tp-21-450

View this article at: https://dx.doi.org/10.21037/tp-21-450 


\section{Introduction}

Premature infants are the key research focus of perinatal medicine. With the development of medical technology, the survival rate of premature infants has been significantly improved, followed by the prevention and treatment of complications (1). Patent ductus arteriosus (PDA) is a common complication in premature infants, which occurs mostly in very low birth weight infants (2). Arterial catheter is an important channel for the flow of fetal circulation from the bifurcation of pulmonary artery to the beginning of left pulmonary artery to the descending aorta. Fullterm newborns within 10-15 hours after birth, due to the contraction of the muscular layer of the arterial catheter wall, the catheter reached functional closure. Premature infants often due to duct wall dysplasia, prostaglandin secretion abnormalities and other reasons, arterial catheter failed to close in time. As a normal pathway between pulmonary artery and aorta in the body, arterial catheter is a necessary pathway for fetal circulation. It can lead to necrotizing enterocolitis, chronic pulmonary disease and congestive heart failure after fetal PDA, which has a direct impact on the survival rate of premature infants and the incidence of sequelae (3). In previous studies, it was found that the use of glucocorticoids in pregnant women can not only reduce the incidence of pulmonary hyaline membrane disease in premature infants, but can also have positive significance for the prevention of PDA, suggesting that adrenal cortex function in premature infants may play a role in the occurrence of PDA (4). B-type brain natriuretic peptide (BNP) is mainly synthesized in ventricular cells and plays an important role in the regulation of extracellular fluid volume (5). Prostacyclin-2 (PGE-2) is an important factor in maintaining the opening of the fetal ductus arteriosus, and the ductus arteriosus can be maintained under the combined action of PGE-2 and prostaglandin E2 (6). There were few studies on the combined diagnosis of cortisol, BNP and PGE-2 in PDA. Based on these factors, this paper analyzed the clinical significance of changes in serum cortisol, BNP, and PGE-2 levels in premature infants with PDA, so as to provide a reference for clinical diagnosis, treatment, and prevention. We present the following article in accordance with the STARD reporting checklist (available at https://dx.doi.org/10.21037/tp-21-450).

\section{Methods}

\section{General information}

A total of 67 premature infants admitted to our hospital from
January 2018 to April 2020 were included, all of whom had PDA (PDA group), including 36 males and 31 females. The gestational age ranged between 28-32 weeks, the average gestational age was $29.11 \pm 0.21$ weeks, the birth weight ranged from 1,000-1,500 g, and the average weight was $1,212.38 \pm 12.26 \mathrm{~g}$. The inclusion criteria were as follows: (I) patients admitted within $24 \mathrm{~h}$ after birth; (II) PDA was diagnosed by echocardiography; (III) complete clinical data; (IV) the family members of the infants were aware of and agreed to this study, and signed informed consent. The exclusion criteria were as follows: (I) congenital diseases or severe asphyxia; (II) severe anemia and pulmonary hypertension were found 1 week after birth; (III) patients with drug allergies. In addition, 62 premature infants born in the same period in our hospital (control group) were selected, who were more than $72 \mathrm{~h}$ after birth, and no PDA, congenital heart disease, infection, or other conditions were confirmed by ultrasound. There were 33 males and 29 females in the control group. The gestational age ranged between 28-32 weeks, and the average gestational age was $29.20 \pm 0.13$ weeks. The birth weight ranged between $1,100-1,500 \mathrm{~g}$, and the average weight was $1,288.38 \pm 12.68 \mathrm{~g}$. There was no difference in general data between the selected subjects $(\mathrm{P}>0.05)$. All procedures performed in this study involving human participants were in accordance with the Declaration of Helsinki (as revised in 2013). The study was approved by Ethics Committee of the First Affiliated Hospital of Xinxiang Medical University (No. EC-021-120) and informed consent was taken from parents or legal guardians of all the patients.

\section{Study methods}

\section{Detection of cortisol, BNP, and PGE-2 levels}

On the 3rd day after birth, $2 \mathrm{~mL}$ peripheral venous blood was collected for examination. Cortisol was detected by an enzyme-linked immunosorbent assay kit (Beckman Coulter, CA, USA). BNP was detected using a fluorescence immunochromatographic assay kit (Easydiagnosis Biomedicine, Wuhan, Hubei). PGE-2 was detected by the double antibody sandwich method and the kit was provided by VICMED Biotechnology Co., Ltd. (Xuzhou, Jiangsu). All procedures were performed in accordance with the reagent instructions.

\section{Ultrasonic examination}

Two-dimensional color pulse Doppler echocardiography with a probe frequency of $2-4 \mathrm{MHz}$ was performed. The ductus arteriosus (DA) diameter, the LA/AO ratio, and the 
Table 1 Comparison of cortisol, BNP, and PGE-2 expression levels in different populations $\left(\bar{x}_{ \pm} \mathrm{S}\right)$

\begin{tabular}{lcccc}
\hline Group & Number & Cortisol (nmol/L) & BNP (ng/L) & PGE-2 (ng/L) \\
\hline Control group & 62 & $22.26 \pm 3.29$ & $70.15 \pm 6.44$ & $23.16 \pm 2.17$ \\
PDA group & 67 & $9.11 \pm 8.26$ & $95.20 \pm 7.42$ & $75.47 \pm 5.19$ \\
$\mathrm{t}$ & - & 11.703 & 20.404 & 73.614 \\
$\mathrm{P}$ & - & $<0.001$ & $<0.001$ & $<0.001$ \\
\hline
\end{tabular}

BNP, B-type brain natriuretic peptide; PGE-2, prostacyclin-2; PDA, patent ductus arteriosus.

Table 2 Comparison of cortisol, BNP, and PGE-2 expression levels in patients with and without symptoms $\left(\bar{x}_{ \pm} \mathrm{S}\right)$

\begin{tabular}{lcccc}
\hline Group & Number & Cortisol (nmol/L) & BNP (ng/L) & PGE-2 (ng/L) \\
\hline Without symptoms & 39 & $13.28 \pm 2.58$ & $81.50 \pm 7.64$ & $69.28 \pm 4.50$ \\
With symptoms & 28 & $7.44 \pm 1.30$ & $103.26 \pm 8.59$ & $82.47 \pm 5.19$ \\
$\mathrm{t}$ & - & 16.415 & 15.155 & 15.366 \\
$\mathrm{P}$ & - & $<0.001$ & $<0.001$ & $<0.001$ \\
\hline
\end{tabular}

BNP, B-type brain natriuretic peptide; PGE-2, prostacyclin-2.

ratio of the ductus arteriosus diameter to the left pulmonary artery diameter (DAD/LPAD) were observed. All indicators needed to be measured over at least 3 cardiac cycles for 3 consecutive measurements. On the 3 rd day after birth, the infants were divided into the asymptomatic group and symptomatic group according to whether there was a dynamic change.

\section{Observation indicators}

(I) Comparison of cortisol, BNP, and PGE-2 expression levels in different populations;

(II) To compare the expression levels of cortisol, BNP, and PGE-2 in infants with and without symptoms;

(III) Analysis of risk factors for PDA in premature infants;

(IV) Analysis of the diagnostic value of cortisol, BNP, and PGE-2 in premature infants with PDA.

\section{Statistical analysis}

SPSS22.0 statistical software (SPSS, Illinois, USA) was used for statistical analysis. The measurement data were expressed as $\bar{x}_{ \pm} \mathrm{S}$. The $t$-test was used for comparisons between 2 groups, and the $F$ test was used for comparisons between groups. The enumeration data were expressed as $\mathrm{n}(\%)$, and the $\chi^{2}$ test was used. Multivariate logistic regression was used to analyze the risk factors of PDA in premature infants. A receiver operating characteristic (ROC) curve was drawn to analyze the diagnostic value of cortisol, BNP, and PGE-2 levels in premature infants with PDA, and the area under the curve (AUC) was calculated. $\mathrm{P}<0.05$ was considered statistically significant.

\section{Results}

Comparison of cortisol, BNP, and PGE-2 expression levels in different populations

The cortisol level in the PDA group was significantly lower than that in the control group, while the BNP and PGE2 levels were significantly higher than those in the control group $(\mathrm{P}<0.05)$, as shown in Table 1 .

\section{Comparison of cortisol, BNP, and PGE-2 expression levels in patients with and without symptoms}

There were 39 cases in the asymptomatic group and 28 cases in the symptomatic group. The level of plasma cortisol in the asymptomatic group was significantly higher than that in the symptomatic group, and the levels of BNP and PGE-2 were significantly lower than those in the symptomatic group $(\mathrm{P}<0.05)$, as shown in Table 2.

\section{Risk factors for PDA in preterm infants}

Logistic regression analysis showed that birth weight $<1,200$ 
Table 3 Analysis of risk factors affecting the prognosis of PDA in premature infants

\begin{tabular}{|c|c|c|c|c|c|c|}
\hline Variable & \multicolumn{3}{|c|}{ Single-factor analysis } & \multicolumn{3}{|c|}{ Multiple-factor analysis } \\
\hline Gestational age ( $\geq 29$ vs. $<29$ weeks) & 1.864 & $0.906-3.835$ & 0.582 & - & - & - \\
\hline Gender (male vs. female) & 1.743 & $0.634-4.793$ & 0.478 & - & - & - \\
\hline Birth weight ( $\geq 1,200$ vs. $<1,200 \mathrm{~g})$ & 1.565 & $1.065-2.298$ & 0.029 & 1.840 & $1.326-2.553$ & $<0.001$ \\
\hline BNP (increase vs. decrease) & 1.838 & $1.413-2.320$ & $<0.001$ & 1.221 & $1.247-1.657$ & 0.038 \\
\hline PGE-2 (increase vs. decrease) & 1.354 & $1.235-1.456$ & 0.031 & 1.617 & $1.127-2.320$ & 0.025 \\
\hline
\end{tabular}

PDA, patent ductus arteriosus; BNP, B-type brain natriuretic peptide; PGE-2, prostacyclin-2.

Table 4 Diagnostic value of cortisol, BNP, and PGE-2 in premature infants with PDA

\begin{tabular}{lcccc}
\hline Predictive factor & AUC & $95 \% \mathrm{Cl}$ & Sensitivity & Specificity \\
\hline Cortisol & 0.719 & $0.593-0.844$ & 0.640 & 0.638 \\
BNP & 0.691 & $0.563-0.819$ & 0.689 & 0.690 \\
PGE-2 & 0.698 & $0.572-0.823$ & 0.699 & 0.658 \\
Cortisol & 0.759 & $0.611-0.859$ & 0.756 & 0.731 \\
+ +BNP+PGE-2 & & & &
\end{tabular}

BNP, B-type brain natriuretic peptide; PGE-2, prostacyclin-2; PDA, patent ductus arteriosus.

$\mathrm{g}$, decreased cortisol, increased BNP, and increased PGE-2 were independent risk factors for PDA in premature infants, and the differences were statistically significant $(\mathrm{P}<0.05)$, as shown in Table 3.

\section{Diagnostic value of cortisol, BNP, and PGE-2 in premature infants with $P D A$}

According to the ROC curve, the sensitivity and specificity of cortisol + BNP + PGE-2 in the diagnosis of PDA in premature infants were $75.60 \%$ and $73.10 \%$, respectively. The AUC value was 0.759 (95\% CI: $0.611-0.859$ ), which was significantly higher than that of the 3 separate tests $(\mathrm{P}<0.05)$, as shown in Table 4 and Figure 1.

\section{Discussion}

BNP is mainly synthesized and secreted by ventricular myocytes. In the process of its secretion or entering the blood circulation, BNP will be divided into biologically active BNP and non-biologically active amino acid B-type

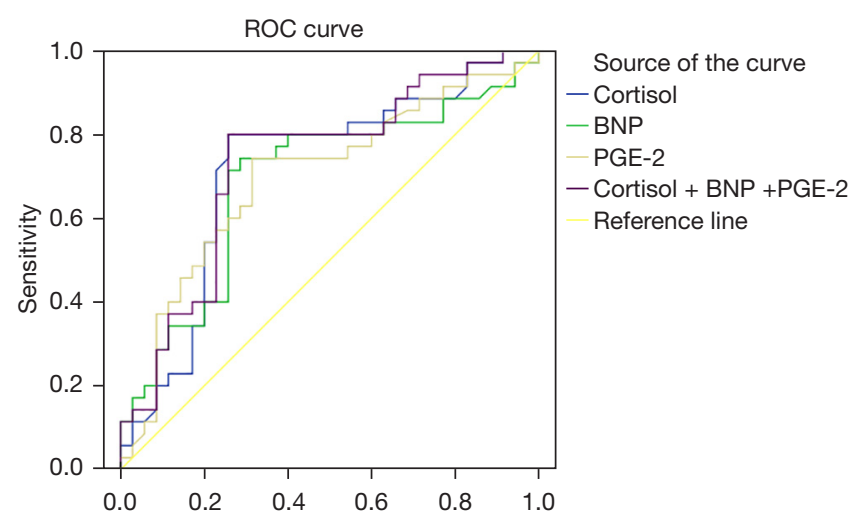

Figure 1 Receiver operating characteristic (ROC) curve of cortisol, B-type brain natriuretic peptide (BNP), and prostacyclin-2 (PGE2) in the single and combined diagnosis of patent ductus arteriosus (PDA) in premature infants.

natriuretic peptide precursor (NT-pro-BNP) by proteolytic enzymes. There is a close relationship between the two, which can reflect the body's pulmonary blood volume and left ventricular load $(7,8)$. Under normal physiological conditions, a small amount of BNP can be detected in the blood circulation. With the increase of ventricular load, myocardial cells can stimulate the secretion of BNP and NT-pro-BNP after stretch stimulation, and increase their expression in the blood (9). After birth, due to the cessation of blood supply to the placenta, the increase of systemic vascular resistance can cause a ventricular pressure increase, resulting in a temporary increase in BNP level. Afterwards, the peripheral vascular resistance is restored to normal (10). In this study, compared with the control group, the BNP level in premature infants with PDA was significantly increased, and the BNP level in the symptomatic group was 
significantly higher than that in the asymptomatic group, suggesting that the change in BNP level can a provide reference for PDA in premature infants.

PGE-2 is synthesized in the vessel wall, which can bind to specific $G$ protein-coupled receptors and activate adenylate cyclase. With the increase of cAMP concentration, it can inhibit the sensitivity of contractile protein calcium ions, thereby relaxing the vessel and maintaining the opening of the vessel (11). The low ability of the lungs to scavenge PGE-2 in premature infants leads to increased PGE2 expression in the blood. The sensitivity of the ductus arteriosus to PGE-2 in premature infants is high, which is one of the causes of delayed closure of the arteries in premature infants $(12,13)$. In this study, the PGE-2 level in premature infants with PDA was significantly higher than that in premature infants without PDA, and the PGE-2 level in the symptomatic group was higher than that in the asymptomatic group, indicating that there was a certain relationship between PGE-2 and PDA.

Cortisol is a stress hormone secreted by the adrenal cortex, which can be affected by infection, asphyxia, and other critical conditions (14). Previous studies used gestational age and prenatal dexamethasone as matching conditions, and found that the level of cortisol was related to the occurrence of $\mathrm{PDA}$ in premature infants $(15,16)$. However, the influence mechanism of cortisol on the closure of the arterial catheter is unclear (17). Animal experiments have shown that cortisol can reduce prostaglandin synthesis by inhibiting phospholipase A, and can reduce the sensitivity of the arterial catheter to the prostaglandin expansion effect $(18,19)$. Studies have also found that cortisol itself has a weak contractile effect on the artery (20). In this study, it was found that the cortisol level in infants with PDA was significantly decreased, and the cortisol level in infants with symptoms was lower.

Through the above, it can be seen that changes in cortisol, BNP, and PGE-2 levels are related to the occurrence and development of PDA. In the analysis of related factors, birth weight $<1,200 \mathrm{~g}$, decreased cortisol, increased BNP, and increased PGE-2 were found to be independent risk factors for PDA in premature infants, which further confirms the relationship between cortisol, BNP, and PGE-2 levels and PDA. It was also found that cortisol, BNP, and PGE-2 have certain diagnostic value for PDA in premature infants, and the combination of the 3 can effectively improve the diagnostic sensitivity, which provides an important reference for the clinical diagnosis of PDA in premature infants.
In conclusion, the expression of cortisol in premature infants with PDA decreased, while the levels of BNP and PGE-2 increased significantly. Dynamic detection of the levels of these 3 factors can provide an important reference for the early diagnosis and progression of PDA.

\section{Acknowledgments}

Funding: None.

\section{Footnote}

Reporting Checklist: The authors have completed the STARD reporting checklist. Available at https://dx.doi. org/10.21037/tp-21-450

Data Sharing Statement: Available at https://dx.doi. org/10.21037/tp-21-450

Conflicts of Interest: All authors have completed the ICMJE uniform disclosure form (available at https://dx.doi. org/10.21037/tp-21-450). The authors have no conflicts of interest to declare.

Ethical Statement: The authors are accountable for all aspects of the work in ensuring that questions related to the accuracy or integrity of any part of the work are appropriately investigated and resolved. All procedures performed in this study involving human participants were in accordance with the Declaration of Helsinki (as revised in 2013). The study was approved by Ethics Committee of the First Affiliated Hospital of Xinxiang Medical University (No.EC-021-120) and informed consent was taken from parents or legal guardians of all the patients.

Open Access Statement: This is an Open Access article distributed in accordance with the Creative Commons Attribution-NonCommercial-NoDerivs 4.0 International License (CC BY-NC-ND 4.0), which permits the noncommercial replication and distribution of the article with the strict proviso that no changes or edits are made and the original work is properly cited (including links to both the formal publication through the relevant DOI and the license). See: https://creativecommons.org/licenses/by-nc-nd/4.0/.

\section{References}

1. Aldana-Aguirre JC, Toye J, Shah PS, et al. Patent ductus 
arteriosus and small for gestational age infants: Treatment approaches and outcomes. Early Hum Dev 2019;131:10-4.

2. Chakraborty A, Philip R, Naik R, et al. Abstract 16551: Transcatheter Closure of Patent Ductus Arteriosus in Premature Infants - How Young is Too Young? Circulation 2020;142:17-9.

3. Rogers LC, Kahn SE, Oeser TH, et al. The Stratus immunofluorometric assay system evaluated for measuring serum cortisol. Clin Chem 1986;32:1588.

4. Landau M. An Interview with George Church. Clin Chem 2020;66:12-24.

5. Markush D, Tsing JC, Gupta S, et al. Fate of the Left Pulmonary Artery and Thoracic Aorta After Transcatheter Patent Ductus Arteriosus Closure in Low Birth Weight Premature Infants. Pediatr Cardiol 2021;42:628-36.

6. Bill R, Carmo LP, Vidondo B, et al. Effect of intramuscular and intravaginal PGE-2 treatment compared to intramuscular oxytocin treatment in eutocic sows on the farrowing performance in a free farrowing system. Theriogenology 2021;161:1-7.

7. Rios DR, Martins FF, El-Khuffash A, et al. Early Role of the Atrial-Level Communication in Premature Infants with Patent Ductus Arteriosus. J Am Soc Echocardiogr 2021;34:423-432.e1.

8. O'Byrne ML, Millenson ME, Grady CB, et al. Trends in transcatheter and operative closure of patent ductus arteriosus in neonatal intensive care units: Analysis of data from the Pediatric Health Information Systems Database. Am Heart J 2019;217:121-30.

9. Philip R, Sathanandam S, Tailor N, et al. A swine model for the premature infant patent ductus arteriosus. J Am Coll Cardiol 2019;73:1686.

10. Rafaeli Rabin R, Rosin I, Matitiau A, et al. Assessing Patent Ductus Arteriosus (PDA) Significance on Cardiac Output by Whole-Body Bio-impedance. Pediatr Cardiol 2020;41:1386-90.

11. McLoughlin RJ, Dacier BM, Hazeltine MD, et al. Intraventricular Hemorrhage and Patent Ductus Arteriosus Ligation Association with Infant Mortality. J

Cite this article as: Cui Q, Liu X, Su G, Zhou C, Wang J. Change and clinical significance of serum cortisol, BNP, and PGE-2 levels in premature infants with patent ductus arteriosus. Transl Pediatr 2021;10(10):2573-2578. doi: 10.21037/tp-21-450
Surg Res 2020;252:192-9.

12. Henry BM, Hsieh WC, Sanna B, et al. Incidence, Risk Factors, and Comorbidities of Vocal Cord Paralysis After Surgical Closure of a Patent Ductus Arteriosus: A Metaanalysis. Pediatr Cardiol 2019;40:116-25.

13. Bennis FC, Andriessen P, van Pul C, et al. Ratio of arterial blood pressures at borders of window surrounding systolic peak indicates patent ductus arteriosus in preterm infants. Physiol Meas 2021;42:015005.

14. Ng SM, Ogundiya A, Didi M, et al. Adrenal function of extremely premature infants in the first 5 days after birth. J Pediatr Endocrinol Metab 2019;32:363-7.

15. Regan W, Benbrik N, Sharma SR, et al. Improved ventilation in premature babies after transcatheter versus surgical closure of patent ductus arteriosus. Int J Cardiol 2020;311:22-7.

16. Kim ES, Kaiser JR, Rios DR, et al. Cerebral Hemodynamics Are Not Affected by the Size of the Patent Ductus Arteriosus. Neonatology 2020;117:182-8.

17. Niccum M, Spyropoulos F, Levin J, et al. Abstract 14185: Targeting Lower Oxygen Saturation Parameters in Premature Infants Does Not Increase Incidence of Pulmonary Hypertension in the Neonatal Intensive Care Unit. Circulation 2020;142:128-9.

18. Usuda K, Kato T, Tsuda T, et al. Impact of sinus rhythm maintenance on major adverse cardiac and cerebrovascular events after catheter ablation of atrial fibrillation: insights from AF frontier ablation registry. Heart Vessels 2021. [Epub ahead of print].

19. Zun Z, Kapse K, Jacobs M, et al. Longitudinal Trajectories of Regional Cerebral Blood Flow in Very Preterm Infants during Third Trimester Ex Utero Development Assessed with MRI. Radiology 2021;299:691-702.

20. Dani C, Lista G, Bianchi S, et al. Intravenous paracetamol in comparison with ibuprofen for the treatment of patent ductus arteriosus in preterm infants: a randomized controlled trial. Eur J Pediatr 2021;180:807-16.

(English Language Editor: C. Betlazar-Maseh) 